\title{
The provision of epidural analgesia during labor according to maternal birthplace: a Norwegian register study
}

\author{
Åsa Henning Waldum ${ }^{1 *}$ D, Anne Flem Jacobsen ${ }^{1,2}$, Mirjam Lukasse ${ }^{3,4}$, Anne Cathrine Staff ${ }^{1,2}$, Ragnhild Sørum Falk ${ }^{5}$,
} Siri Vangen ${ }^{2,6}$ and Ingvil Krarup Sørbye $e^{1,6}$

\begin{abstract}
Background: The provision of epidural analgesia during labor is ideally a shared decision between the woman and her health care provider. However, immigrant characteristics such as maternal birthplace could affect decisionmaking and thus access to pain relief. We aimed to assess disparities in the provision of epidural analgesia in planned vaginal birth according to maternal region of birth.

Methods: We performed a nation-wide register study of 842,496 live-born singleton deliveries in Norway between 2000 and 2015. Maternal birthplace was categorized according to the Global Burden of Disease framework. The provision of epidural analgesia was compared in regression models stratified by parity and mode of delivery.

Results: Compared to native-born women, primiparous women from Latin America/Caribbean countries with an instrumental vaginal delivery were most likely to be provided epidural analgesia (OR 2.12,95\%Cl 1.69-2.66), whilst multiparous women from Sub-Saharan Africa with a spontaneous vaginal delivery were least likely to be provided epidural analgesia (OR 0.42, 95\% C 0.39-0.44). Longer residence time was associated with a higher likelihood of being provided analgesia, whereas effects of maternal education varied by Global Burden of Disease group.

Conclusions: Disparities in the likelihood of being provided epidural analgesia were observed by maternal birthplace. Further studies are needed to consider whether the identified disparities represent women's own preferences or if they are the result of heterogeneous access to analgesia during labor.
\end{abstract}

Keywords: Analgesia obstetric, Delivery obstetric, Immigration, Socioeconomic factors, Decision making

\section{Background}

Women have always sought to relieve pain during labor. The most common methods of analgesia during labor in high-income settings are regional analgesic methods [1]. These include both epidural analgesia, primarily initiated in the first stage of labor; and pudendal analgesia, provided during the second stage of labor [2]. Epidural analgesia is by far the most common method. The provision

\footnotetext{
* Correspondence: awaldum@ous-hf.no

'Division of Obstetrics and Gynaecology, Oslo University Hospital, Sognsvannsveien 20, 0372 Oslo, Norway

Full list of author information is available at the end of the article
}

of analgesia is associated with a positive childbirth experience [3]. However, several factors might influence the need for labor analgesia. Primiparous women and women undergoing instrumental vaginal delivery (vacuum and/or forceps extraction) have an increased need for analgesia compared to multiparous women and women giving birth spontaneously [4].

Ideally, the provision of pain relief during delivery is a shared decision between the healthcare professional and the woman $[1,5]$. Shared decision-making includes the patient's preferences and the healthcare professional's knowledge about the benefits and risks of each option

C C The Author(s). 2020 Open Access This article is licensed under a Creative Commons Attribution 4.0 International License, which permits use, sharing, adaptation, distribution and reproduction in any medium or format, as long as you give appropriate credit to the original author(s) and the source, provide a link to the Creative Commons licence, and indicate if changes were made. The images or other third party material in this article are included in the article's Creative Commons licence, unless indicated otherwise in a credit line to the material. If material is not included in the article's Creative Commons licence and your intended use is not permitted by statutory regulation or exceeds the permitted use, you will need to obtain permission directly from the copyright holder. To view a copy of this licence, visit http://creativecommons.org/licenses/by/4.0/ The Creative Commons Public Domain Dedication waiver (http://creativecommons.org/publicdomain/zero/1.0/) applies to the data made available in this article, unless otherwise stated in a credit line to the data. 
[5-7]. Health system characteristics, for example the level of training and skills in obstetrics, may influence the health care staff's provision of analgesia. The provision of regional analgesic methods during labor requires more information and cooperation between the health care staff and the woman, compared to other types of pain relief [8]. In contrast, pain relief in a planned cesarean delivery is not prone to a shared decision, as protocols are mainly standardized.

Women in reproductive age across most European countries are becoming more ethnically diverse [9]. Previous studies have shown an increased risk of substandard care and poor maternal and neonatal outcome among certain immigrant groups [10-13]. Although women's desires for pain relief vary, a shared decision might be affected by immigrant characteristics. The woman's birthplace, health literacy, and residence time in a new country could affect the rate of analgesia during labor. A few Norwegian studies have explored the association between maternal birthplace and the provision of epidural analgesia. These have suggested that Somali and Pakistani migrant women were provided less pain relief during labor than women without migration background. Little is known about women from other regions of the world $[8,10]$. Thus, in this study, we aimed to assess the provision of epidural analgesia according to maternal birthplace during planned vaginal birth. Norway is a suitable setting for assessing disparities in epidural analgesia, due to a national compulsory registration of all deliveries and a universal public health care system including free of charge pregnancy ante- and perinatal care.

\section{Methods}

\section{Study design and population}

In Norway most childbirths take place inside the public health care system at hospitals and very few women give birth at home. We linked data from the Medical Birth Register of Norway (MBRN) [14, 15] to information from Statistics Norway about maternal birthplace, maternal education, year of arrival to Norway and reason for immigration (for immigrants only). The MBRN is a national registry with mandatory reporting of all pregnancies ending after 12 complete weeks of gestation. We initially extracted information on all vaginal births in Norway between January 2000 and December 2015. We excluded neonates with a birth weight $<500 \mathrm{~g}$ and/or gestational age $<23$ weeks. To avoid misclassifications, we excluded deliveries with $\mathrm{z}$-scores (birth weight for gestational week and sex) $> \pm 4$ standard deviations. We also excluded abortions and intrauterine fetal death, births with missing maternal birthplace, multiple births and births outside institutions as these women were not eligible for analgesia. Finally, we excluded planned cesarean deliveries, as analgesia is routinely given to these women during surgery (Supplementary Flowchart).

\section{Variables}

The primary outcome was the provision of epidural analgesia. The MBRN registers all types of analgesia provided during labor. Each woman could utilize more than one method of analgesia. The case reporting form contains of tick-boxes for each type of analgesia, which the attending midwife fills out after delivery.

The main exposure was the mother's own birthplace, as registered by Statistics Norway. For immigrants, the mother's country of birth was categorized into region of birth according to the Global Burden of Disease (GBD) framework [16]. All high-income countries were grouped into the category "high-income countries". Furthermore, middle- and low-income countries were categorized into the following GBD regions: "Europe/Central Asia", "Sub-Saharan Africa", "North Africa/Middle East", "South Asia", "East Asia/Pacific", or "Latin America/Caribbean". Norwegian-born women were categorized as "native-born".

Independent variables were pre-defined and selected based on their potential association with the outcome and exposure according to previous literature. Maternal age at delivery was categorized as $<20,20-34,35-40$ or $\geq 40$ years. Marital status was categorized as married/cohabiting or not. Paternal birthplace was categorized as nativeborn or not. Maternal education was defined as completed years of education and categorized as lower ( $\leq 10$ years), middle (11-13 years), or higher ( $>13$ years). Year of delivery was categorized into 4 periods (2000-2003, 20042007, 2008-2011 or 2012-2015). For immigrants we further included maternal residence time in Norway by subtracting the year of first arrival in Norway from the year of delivery, and categorized residence time as $<2$ years, $2-10$ years, or $>10$ years, and reason for immigration (refugee, labor/education, family reunification or other).

Parity was dichotomized as primiparous or multiparous. Mode of delivery was categorized as spontaneous, instrumental (forceps and/or vacuum extraction) or emergency cesarean delivery. Previous cesarean delivery was noted for the multiparous women. Gestational age was categorized as <37 weeks, 37-41 weeks, or $\geq 42$ completed weeks and birth weight into $<2500 \mathrm{~g}(\mathrm{~g}), 2500-3999 \mathrm{~g}$, or $\geq 4000$ g. Epidural, spinal and pudendal analgesia were noted as utilized $(\mathrm{y} / \mathrm{n})$, as were nitrous oxide, local anesthetics and opiates. The size of the obstetric department was pre-categorized by the MBRN as numbers of births per year $(1-499,500-1499,1500-2999$, or $\geq 3000)$.

\section{Statistical analyses}

Descriptive statistics are presented as frequencies and proportions according to maternal birthplace. The 
provision of epidural analgesia is presented as proportions within each group of maternal birthplaces stratified by mode of delivery and parity.

The association between epidural analgesia and birthplace was investigated with logistic regression analyses. Women born in Norway were defined as the reference group. Both crude and adjusted odds ratios (OR) with 95\% confidence intervals (CI) are presented. Adjustments were made for maternal age at delivery, marital status, education, birth weight, year of childbirth, and size of obstetric department. Gestational age was not included in the regression analyses due to its strong correlation to birth weight. For multiparous women, we adjusted for number of previous births and previous cesarean delivery.

We performed stratified analyses by mode of delivery. Since there was an interaction between maternal birthplace and parity, we also stratified for parity. Further, interaction between maternal birthplace and the adjusting variables were explored by entering the interaction terms, one at a time, into the model. Interactions with $p<0.001$ are reported in the text and presented graphically.

We performed sensitivity analysis to explore the impact of residence time in Norway, where we included residence time in the regression models. In these analyses, we used women born in high-income countries as reference.

Due to the large sample size, we considered an association with p-value of $<.001$ as statistically significant. We conducted all analyses using SPSS version 25 (IBM Corp., Armonk, NY, USA) and Stata (StataCorp. 2017. Stata Statistical Software: Release 15. College Station, TX: StatCorp LLC).

\section{Ethics approval and consent to participate}

The study was approved by the Regional Committees for Medical and Health Research Ethics South East Norway in 2017 (reference 2016/417/REK) including waiver of participant's individual consent.

\section{Results}

The final study population included 842,496 deliveries during the 16-year period. Maternal demographic and obstetric characteristics by maternal birthplace are presented in Tables 1 and 2. Immigrant women accounted for $21 \%$ of the births $(n=175,038)$. The two largest immigrant groups included women born in high-income countries and Europe/Central Asia, whilst the smallest immigrant group included women born in Latin America/Caribbean (3\%) (Supplementary Table 1). Compared to native-born women, a higher proportion of immigrants had lower education, as well as a previous cesarean delivery. The Sub-Saharan African group had the lowest proportion of primiparous women (31.6\%). The large obstetric departments had the highest rate of immigrant deliveries. The number of deliveries increased over the study years for all immigrant groups in contrast to the declining birth number in the native-born (in Norway) group. Residence time and reasons for migration varied between GBD groups, and according to known historic migration patterns (Table 1). The proportion of instrumental vaginal delivery was highest among women from East Asia/Pacific (12.4\%) compared to $9.4 \%$ among native-born women, while women from Sub-Saharan Africa had $17.3 \%$ emergency cesarean deliveries compared to $9.5 \%$ among native-born women. SubSaharan African women had the highest proportion on pregnancies $\geq 42$ weeks of gestational age while nativeborn women had the highest proportion of newborn weighing $\geq 4000 \mathrm{~g}$. Nitrous oxide was the most common analgesia method (40.9\%), followed by local anesthesia $(30.8 \%)$, epidural analgesia (30.0\%), spinal analgesia (6.4\%), opiates (4.7\%), and pudendal analgesia (2.4\%) (Table 2).

Across all GBD groups, primiparous women were - as expected - provided epidural analgesia more often, when compared to multiparous women (Table 3). Similarly, women with instrumental delivery were more often provided epidural analgesia compared to women with spontaneous delivery. The lowest provision of epidural analgesia was observed among multiparous women from Sub-Saharan Africa delivering spontaneously (9\%). The highest provision of epidural analgesia was observed in primiparous Latin America/Caribbean women with an instrumental vaginal delivery (78\%). Among women delivered with emergency cesarean section, women from Latin America/Caribbean had the highest epidural analgesia rate (Table 3 ).

The regression analysis showed heterogeneity in the likelihood of providing epidural analgesia by maternal birthplace. Primiparous women with spontaneous and instrumental vaginal delivery and women born in SubSaharan Africa and East Asia/Pacific had lower odds of being provided epidural analgesia compared to nativeborn women. Women from Latin America/Caribbean were more likely to be provided analgesia. Women born in Sub-Saharan Africa were least likely to be provided epidural analgesia, if they were subsequently delivered by emergency cesarean section, while this was the opposite in women born in Latin America/Caribbean. For multiparous women in all modes of delivery, women from Sub-Saharan Africa had the lowest odds of being provided epidural analgesia, while women from Latin America/Caribbean had the highest odds (Table 4). In addition, we observed a linear effect of time during the study period; the provision of epidural analgesia increased by $3.5-5 \%$ each year. 
Table 1 Demographic characteristics by maternal birthplace, $n=842,496$ women

\begin{tabular}{|c|c|c|c|c|c|c|c|c|c|c|c|c|c|c|c|c|}
\hline & \multirow{2}{*}{\multicolumn{2}{|c|}{$\begin{array}{l}\text { Native-born } \\
\text { Norway }\end{array}$}} & \multicolumn{14}{|c|}{ Immigrants } \\
\hline & & & \multicolumn{2}{|c|}{ High-income } & \multicolumn{2}{|c|}{ Europe/Central Asia } & \multicolumn{2}{|c|}{$\begin{array}{l}\text { Sub-Saharan } \\
\text { Africa } \\
\end{array}$} & \multicolumn{2}{|c|}{$\begin{array}{l}\text { North Africa/ } \\
\text { Middle East } \\
\end{array}$} & \multicolumn{2}{|c|}{ South Asia } & \multicolumn{2}{|c|}{$\begin{array}{l}\text { East Asia/ } \\
\text { Pacific } \\
\end{array}$} & \multicolumn{2}{|c|}{$\begin{array}{l}\text { Latin America/ } \\
\text { Caribbean }\end{array}$} \\
\hline & $n$ & $\%$ & $n$ & $\%$ & $n$ & $\%$ & $n$ & $\%$ & $n$ & $\%$ & $n$ & $\%$ & $n$ & $\%$ & $n$ & $\%$ \\
\hline & 667,458 & 79.2 & 41,450 & 4.9 & 41,185 & 4.9 & 23,499 & 2.8 & 26,735 & 3.2 & 22,585 & 1.4 & 25,107 & 3.0 & 5477 & 0.7 \\
\hline \multicolumn{17}{|l|}{ Maternal age (years) } \\
\hline$<20$ & 15,358 & 2.3 & 414 & 1.0 & 693 & 1.7 & 594 & 2.5 & 658 & 2.5 & 127 & 1.1 & 280 & 1.1 & 167 & 3.0 \\
\hline $20-34$ & 540,595 & 81.0 & 30,545 & 73.7 & 34,646 & 84.1 & 18,692 & 79.5 & 21,556 & 80.6 & 9974 & 86.1 & 20,052 & 79.9 & 4207 & 76.8 \\
\hline $35-40$ & 102,249 & 15.3 & 9487 & 22.9 & 5273 & 12.8 & 3637 & 15.5 & 3946 & 14.8 & 1330 & 11.5 & 4278 & 17.0 & 992 & 18.1 \\
\hline$>40$ & 9256 & 1.4 & 1004 & 2.4 & 573 & 1.4 & 576 & 2.5 & 575 & 2.2 & 154 & 1.3 & 497 & 2.0 & 111 & 2.0 \\
\hline Married/Cohabiting & 618,042 & 92.6 & 39,156 & 94.5 & 39,115 & 95.0 & 17,744 & 75.5 & 25,107 & 93.9 & 11,113 & 95.9 & 23,284 & 92.8 & 4899 & 89.4 \\
\hline \multicolumn{17}{|l|}{ Paternal birthplace } \\
\hline Native-born & 609,302 & 91.3 & 27,540 & 66.4 & 9612 & 23.3 & 2253 & 9.6 & 1532 & 5.7 & 2553 & 22.0 & 12,281 & 48.9 & 4266 & 77.9 \\
\hline Immigrant & 49,586 & 7.4 & 13,090 & 31.6 & 30,321 & 73.6 & 17,689 & 75.3 & 24,014 & 89.8 & 8775 & 75.7 & 12,132 & 48.3 & 1061 & 19.4 \\
\hline Missing & 8570 & 1.3 & 820 & 2.0 & 1252 & 3.0 & 3557 & 15.1 & 1189 & 4.4 & 257 & 2.2 & 694 & 2.8 & 150 & 2.7 \\
\hline \multicolumn{17}{|c|}{ Maternal education (years) } \\
\hline Lower $(\leq 10)$ & 92,166 & 13.8 & 3723 & 9.0 & 6630 & 16.1 & 9944 & 42.3 & 10,215 & 38.2 & 3971 & 34.3 & 7644 & 30.4 & 1331 & 24.3 \\
\hline Middle (11-13) & 209,538 & 31.4 & 8580 & 20.7 & 9161 & 22.2 & 3745 & 15.9 & 4786 & 17.9 & 2089 & 18.0 & 5617 & 22.4 & 1239 & 22.6 \\
\hline Higher (> 13) & 364,809 & 54.7 & 23,751 & 57.3 & 16,921 & 41.1 & 3267 & 13.9 & 5047 & 18.9 & 2915 & 25.2 & 7550 & 30.1 & 2019 & 36.9 \\
\hline missing & 945 & 0.1 & 5396 & 13.0 & 8473 & 20.6 & 6543 & 27.8 & 6687 & 25.0 & 2610 & 22.5 & 4296 & 17.1 & 888 & 16.2 \\
\hline \multicolumn{17}{|c|}{ Size of obstetric department (births/year) } \\
\hline $1-499$ & 74,040 & 11.1 & 2912 & 7.0 & 3806 & 9.2 & 1865 & 7.9 & 1452 & 5.4 & 182 & 1.6 & 1923 & 7.7 & 389 & 7.1 \\
\hline $500-1499$ & 155,308 & 23.3 & 6187 & 14.9 & 7507 & 18.2 & 3473 & 14.8 & 3023 & 11.3 & 443 & 3.8 & 3954 & 15.7 & 906 & 16.5 \\
\hline 1500-2999 & 175,128 & 26.2 & 12,710 & 30.7 & 12,053 & 29.3 & 6106 & 26.0 & 8213 & 30.7 & 2825 & 24.4 & 6400 & 25.5 & 1484 & 27.1 \\
\hline$\geq 3000$ & 262,982 & 39.4 & 19,641 & 47.4 & 17,819 & 43.3 & 12,055 & 51.3 & 14,047 & 52.5 & 8135 & 70.2 & 12,830 & 51.1 & 2698 & 49.3 \\
\hline \multicolumn{17}{|l|}{ Year of delivery } \\
\hline 2000-2003 & 161,292 & 26.6 & 18,908 & 21.2 & 4949 & 11.2 & 3393 & 13.7 & 5193 & 18.0 & 3109 & 20.6 & 5387 & 19.7 & 943 & 15.4 \\
\hline 2004-2007 & 155,216 & 25.6 & 20,784 & 23.3 & 7092 & 16.0 & 5087 & 20.5 & 6743 & 23.4 & 3613 & 23.9 & 6236 & 22.8 & 1347 & 22.0 \\
\hline 2008-2011 & 153,243 & 25.2 & 24,158 & 27.1 & 13,079 & 29.6 & 7330 & 29.6 & 7889 & 27.4 & 4005 & 26.5 & 7283 & 26.7 & 1823 & 29.8 \\
\hline 2012-2015 & 137,390 & 22.6 & 25,173 & 28.3 & 19,095 & 43.2 & 8965 & 36.2 & 8964 & 31.1 & 4393 & 29.1 & 8406 & 30.8 & 2008 & 32.8 \\
\hline \multicolumn{17}{|c|}{ Residence time (years) ${ }^{1}$} \\
\hline$<2$ & & & 5124 & 12.4 & 12,095 & 29.4 & 5928 & 25.2 & 6926 & 25.9 & 2037 & 17.6 & 5321 & 21.2 & 1173 & 21.4 \\
\hline $2-10$ & & & 19,847 & 47.9 & 23,709 & 57.6 & 13,786 & 58.7 & 14,245 & 53.3 & 5334 & 46.0 & 13,655 & 54.4 & 2507 & 45.8 \\
\hline$>10$ & & & 6142 & 14.8 & 5281 & 12.8 & 3216 & 13.7 & 5475 & 20.5 & 3641 & 31.4 & 5452 & 21.7 & 569 & 10.4 \\
\hline Missing & & & 10,337 & 24.9 & 100 & 0.2 & 569 & 2.4 & 89 & 0.3 & 573 & 5.0 & 679 & 2.7 & 1228 & 22.4 \\
\hline \multicolumn{17}{|c|}{ Reason for immigration } \\
\hline Refugee & & & 101 & 0.2 & 6621 & 16.1 & 11,784 & 50.2 & 5786 & 21.6 & 186 & 1.6 & 1662 & 6.6 & 93 & 1.7 \\
\hline Labor/education & & & 6703 & 16.2 & 15,056 & 36.6 & 952 & 4.1 & 354 & 1.3 & 428 & 3.7 & 3195 & 12.7 & 613 & 11.2 \\
\hline Family reunification & & & 6529 & 15.8 & 18,199 & 44.2 & 9425 & 40.1 & 18,295 & 68.4 & 7938 & 68.5 & 16,718 & 66.6 & 3304 & 60.3 \\
\hline Other & & & 12,549 & 30.3 & 274 & 0.7 & 252 & 1.1 & 253 & 1.0 & 99 & 0.9 & 168 & 0.7 & 49 & 0.9 \\
\hline Missing & & & 15,568 & 37.6 & 1035 & 2.5 & 1086 & 4.6 & 2047 & 7.7 & 2934 & 25.3 & 3364 & 13.4 & 1418 & 25.9 \\
\hline
\end{tabular}

${ }^{1}$ Immigrants only

Maternal education modified the effect of maternal birthplace on the likelihood of being provided epidural analgesia. Among native-born women, those with higher education were less likely to be provided epidural analgesia compared to those with lower education. Conversely, among several immigrant groups from mediumor low-income countries (Europe/Central Asia, SubSaharan Africa, North Africa/Middle East, South Asia, 
Table 2 Obstetric characteristics by maternal birthplace, $n=842,496$ women

\begin{tabular}{|c|c|c|c|c|c|c|c|c|c|c|c|c|c|c|c|c|}
\hline & \multirow{2}{*}{\multicolumn{2}{|c|}{$\frac{\text { Native-born }}{\text { Norway }}$}} & \multicolumn{14}{|c|}{ Immigrants } \\
\hline & & & \multicolumn{2}{|c|}{ High-income } & \multicolumn{2}{|c|}{$\begin{array}{l}\text { Europe/ } \\
\text { Central Asia }\end{array}$} & \multicolumn{2}{|c|}{$\begin{array}{l}\text { Sub-Saharan } \\
\text { Africa }\end{array}$} & \multicolumn{2}{|c|}{$\begin{array}{l}\text { North Africa/ } \\
\text { Middle East }\end{array}$} & \multicolumn{2}{|c|}{ South Asia } & \multicolumn{2}{|c|}{$\begin{array}{l}\text { East Asia/ } \\
\text { Pacific }\end{array}$} & \multicolumn{2}{|c|}{$\begin{array}{l}\text { Latin America/ } \\
\text { Caribbean }\end{array}$} \\
\hline & $n$ & $\%$ & $n$ & $\%$ & $n$ & $\%$ & $n$ & $\%$ & $n$ & $\%$ & $n$ & $\%$ & $n$ & $\%$ & $n$ & $\%$ \\
\hline & 667,458 & 79.2 & 41,450 & 4.9 & 41,185 & 4.9 & 23,499 & 2.8 & 26,735 & 3.2 & 22,585 & 1.4 & 25,107 & 3.0 & 5477 & 0.7 \\
\hline Primiparous & 286,766 & 43.0 & 19,082 & 46.0 & 20,468 & 49.7 & 7434 & 31.6 & 9751 & 36.5 & 4148 & 35.8 & 11,223 & 44.7 & 2778 & 50.7 \\
\hline \multicolumn{17}{|l|}{ Mode of delivery } \\
\hline Spontaneous vaginal & 541,515 & 81.1 & 32,118 & 77.5 & 32,343 & 78.5 & 17,391 & 74.0 & 21,222 & 79.4 & 9007 & 77.7 & 18,173 & 72.4 & 3927 & 71.7 \\
\hline Instrumental vaginal & 62,722 & 9.4 & 4761 & 11.5 & 4750 & 11.5 & 2037 & 8.7 & 2627 & 9.8 & 1212 & 10.5 & 3116 & 12.4 & 589 & 10.8 \\
\hline Emergency cesarean section & 63,221 & 9.5 & 4571 & 11.0 & 4092 & 9.9 & 4071 & 17.3 & 2886 & 10.8 & 1366 & 11.8 & 3818 & 15.2 & 961 & 15.7 \\
\hline Previous cesarean delivery ${ }^{1}$ & 42,145 & 11.1 & 2759 & 12.3 & 2295 & 11.1 & 2833 & 17.6 & 2158 & 12.7 & 1229 & 16.5 & 1978 & 14.2 & 528 & 19.6 \\
\hline \multicolumn{17}{|l|}{ Gestational age (weeks) } \\
\hline$<37$ & 33,335 & 5.0 & 1928 & 4.7 & 1755 & 4.3 & 1144 & 4.9 & 1332 & 5.0 & 769 & 6.6 & 1724 & 6.9 & 305 & 5.6 \\
\hline $37-41$ & 590,202 & 88.4 & 36,913 & 89.1 & 36,920 & 89.6 & 19,996 & 85.1 & 24,063 & 90.0 & 10,361 & 89.4 & 22,649 & 90.2 & 4876 & 89.0 \\
\hline$\geq 42$ & 43,921 & 6.6 & 2609 & 6.3 & 2510 & 6.1 & 2359 & 10.0 & 1340 & 5.0 & 455 & 3.9 & 734 & 2.9 & 296 & 5.4 \\
\hline \multicolumn{17}{|l|}{ Birth weight (gram) } \\
\hline$<2500$ & 21,084 & 3.2 & 1280 & 3.1 & 1170 & 2.8 & 1100 & 4.7 & 1021 & 3.8 & 786 & 6.8 & 1162 & 4.6 & 212 & 3.9 \\
\hline 2500-3999 & 504,827 & 75.6 & 32,584 & 78.6 & 32,992 & 80.1 & 19,437 & 82.7 & 22,489 & $84-1$ & 9962 & 86.0 & 21,408 & 85.3 & 4525 & 82.6 \\
\hline$\geq 4000$ & 141,547 & 21.2 & 7586 & 18.3 & 7023 & 17.1 & 2962 & 12.6 & 3225 & 12.1 & 837 & 7.2 & 2537 & 10.1 & 740 & 13.5 \\
\hline Epidural analgesia $^{2}$ & 199,822 & 29.9 & 13,970 & 33.7 & 13,898 & 33.7 & 5161 & 22.0 & 7267 & 27.2 & 3188 & 27.5 & 6755 & 26.9 & 2498 & 45.6 \\
\hline Spinal analgesia ${ }^{2}$ & 41,312 & 6.2 & 2794 & 6.7 & 2451 & 6.0 & 2289 & 9.7 & 1742 & 6.5 & 829 & 7.2 & 2202 & 8.8 & 519 & 9.5 \\
\hline Pudendal analgesia ${ }^{2}$ & 16,379 & 2.5 & 1179 & 2.8 & 908 & 2.2 & 383 & 1.6 & 497 & 1.9 & 240 & 2.1 & 566 & 2.3 & 144 & 2.6 \\
\hline Nitrous Oxide ${ }^{2}$ & 281,531 & 42.2 & 16,161 & 39.0 & 16,131 & 39.2 & 7569 & 32.2 & 9161 & 34.3 & 3496 & 30.2 & 8611 & 34.3 & 2110 & 38.5 \\
\hline Local anesthesia ${ }^{2}$ & 207,465 & 31.1 & 12,852 & 31.0 & 11,981 & 29.1 & 6449 & 27.4 & 7052 & 26.4 & 3696 & 31.9 & 8231 & 32.8 & 1442 & 26.3 \\
\hline Opiates $^{2}$ & 32,861 & 4.9 & 1628 & 3.9 & 1264 & 3.1 & 659 & 2.8 & 1285 & 4.8 & 517 & 4.5 & 1113 & 4.4 & 203 & 3.7 \\
\hline
\end{tabular}

${ }^{1}$ Multiparous women only. ${ }^{2}$ Multiple analgesia modalities may have been used

or East Asia/Pacific), those with higher education were more likely to be provided epidural analgesia compared to those with lower education (Fig. 1).

However, within education categories, the absolute likelihood of being provided epidural analgesia was lower among the majority of immigrants, compared to nativeborn women. Furthermore, among multiparous women who delivered spontaneously, interaction effects were observed between maternal birthplace and parity and between maternal birthplace and year of childbirth. Among women from Europe/Central Asia, Sub-Saharan Africa, North Africa/Middle East, South Asia, and East Asia/Pacific, the provision of epidural analgesia decreased by increasing parity, while in the other groups, the provision did not differ by number of deliveries (data not shown). For the same immigrant groups, the increasing provision of epidural analgesia by calendar time was more pronounced than in the remaining groups (data not shown). Only one interaction effect was observed among women with emergency cesarean section; previous cesarean section reduced the risk of epidural analgesia in women from East Asia/Pacific.
In sensitivity analyses among immigrants only, residence time slightly affected the association between maternal birthplace and provision of epidural analgesia. Among primiparous women with an instrumental delivery, provision of epidural analgesia was increased by the residence time in Norway (Supplementary Table 2).

Finally, we found an almost $50 \%$ reduction of preeclampsia, but stable rates of diabetes over the time period (Supplementary Table 3). Sub analyses for spinal analgesia and pudendal block revealed the same direction of effect (Supplementary Table 4 and 5).

\section{Discussion}

\section{Summary of evidence}

This study, using population-based data from the Norwegian Birth Registry shows that there is a globally significant effect of birthplace on the use of epidural analgesia. We demonstrated some disparities in the provision of epidural analgesia by maternal birthplace. Immigrants from Latin America/Caribbean were consistently more likely to be provided epidural analgesia compared to native-born women. In contrast, the provision 
Table 3 Epidural analgesia provision by maternal birthplace, mode of delivery and parity

\begin{tabular}{|c|c|c|c|c|c|c|c|c|c|}
\hline & \multicolumn{3}{|c|}{$\begin{array}{l}\text { Spontaneous Vaginal Delivery } \\
\text { Epidural analgesia }\end{array}$} & \multicolumn{3}{|c|}{$\begin{array}{l}\text { Instrumental Vaginal Delivery* } \\
\text { Epidural analgesia }\end{array}$} & \multicolumn{3}{|c|}{$\begin{array}{l}\text { Emergency Cesarean Delivery } \\
\text { Epidural analgesia }\end{array}$} \\
\hline & $n$ & $\%$ & $95 \% \mathrm{Cl}$ & $n$ & $\%$ & $95 \% \mathrm{Cl}$ & $\mathrm{n}$ & $\%$ & $95 \% \mathrm{Cl}$ \\
\hline Primiparous & 94,093 & 37.8 & $37.6-38.0$ & 38,580 & 61.7 & $61.3-62.0$ & 27,126 & 53.8 & $53.4-54.2$ \\
\hline Norway & 75,389 & 37.6 & $37.4-37.8$ & 29,556 & 61.1 & $60.7-61.5$ & 20,199 & 53.4 & $52.9-53.9$ \\
\hline High-income & 4973 & 40.0 & $39.1-40.9$ & 2392 & 64.8 & $63.2-66.3$ & 1729 & 58.5 & $56.7-60.2$ \\
\hline Europe / Central Asia & 5777 & 41.2 & $40.4-42.0$ & 2458 & 65.2 & $63.7-66.8$ & 1546 & 57.7 & $55.8-59.6$ \\
\hline Sub-Saharan Africa & 1418 & 33.8 & $32.4-35.3$ & 734 & 57.5 & $54.8-60.3$ & 937 & 47.7 & $45.5-50.0$ \\
\hline North Africa / Middle East & 2541 & 39.4 & $38.2-40.6$ & 1230 & 66.5 & $64.3-68.7$ & 741 & 51.2 & $48.6-53.8$ \\
\hline South Asia & 998 & 38.1 & $36.2-40.0$ & 555 & 65.6 & $62.3-68.8$ & 326 & 47.9 & $44.1-51.8$ \\
\hline East Asia /Pacific & 2085 & 31.0 & $29.9-32.2$ & 1298 & 56.6 & $54.5-58.6$ & 1232 & 55.6 & $53.5-57.7$ \\
\hline Latin America/ Caribbean & 912 & 53.8 & $51.4-56.2$ & 357 & 78.3 & $74.2-82.0$ & 416 & 66.5 & $62.6-70.1$ \\
\hline Multiparous & 72,612 & 17.0 & $16.9-17.1$ & 9598 & 49.9 & $49.2-50.6$ & 10,550 & 30.5 & $30.0-31.0$ \\
\hline Norway & 59,723 & 17.5 & $17.4-17.6$ & 7256 & 50.6 & $49.8-51.4$ & 7699 & 30.4 & $29.8-30.9$ \\
\hline High-income & 3716 & 18.9 & $18.3-19.4$ & 599 & 56.1 & $53.0-59.1$ & 561 & 34.8 & $32.5-37.2$ \\
\hline Europe / Central Asia & 3104 & 16.9 & $16.4-17.5$ & 518 & 52.7 & $49.6-55.9$ & 495 & 35.0 & $32.5-37.6$ \\
\hline Sub-Saharan Africa & 1224 & 9.3 & $8.8-9.8$ & 279 & 36.7 & $33.2-40.2$ & 569 & 27.0 & $25.1-28.9$ \\
\hline North Africa / Middle East & 2020 & 13.7 & $13.1-14.2$ & 356 & 45.8 & $42.2-49.3$ & 379 & 26.3 & $24.1-28.7$ \\
\hline South Asia & 942 & 14.8 & 13.9-15.6 & 176 & 48.1 & $42.9-53.3$ & 191 & 27.8 & $24.5-31.4$ \\
\hline East Asia /Pacific & 1282 & 11.2 & $10.6-11.8$ & 328 & 39.9 & $36.5-43.3$ & 530 & 33.0 & $30.7-35.4$ \\
\hline Latin America /Caribbean & 601 & 26.9 & $25.1-28.8$ & 86 & 64.7 & $55.9-72.7$ & 126 & 37.6 & $32.4-43.0$ \\
\hline
\end{tabular}

$\mathrm{Cl}$ confidence interval *includes deliveries by vacuum and/or forceps extraction

of epidural analgesia in immigrants born in low- and middle-income countries varied across maternal birthplace. Compared to native-born women, women born in Sub-Saharan Africa or East Asia/Pacific were less likely to be provided epidural analgesia. Longer residence time in Norway was associated with a higher likelihood of being provided analgesia, whereas effects of maternal education depended on GBD group.

Our results for Sub-Saharan women in Norway confirms findings from Bakken et al. (2015) of the low provision of epidural analgesia among Somali-born immigrants, the largest migrant group from Sub-Saharan Africa in Norway [10]. Regarding South Asian women, we found that primiparous women had similar chances of being provided epidural analgesia as native-born women, whereas multiparous women had a reduced likelihood. This is in line with a previous Norwegian study by Vangen et al. [8], where Pakistani-born women were found to be provided less epidural analgesia regardless of parity.

Our results are likely to have multicausal explanations. Firstly, our result could be influenced by real differences in women's own wishes and needs. Cultural norms and perceptions of labor pain as well as knowledge of side effects of pain relief could affect women's choices, even though women's prenatal analgesia preference does not always match their actual use [17]. However, we found significant variation also in women exposed to an instrumental vaginal delivery, where pain relief is strongly advised by the midwife and obstetrician. Overall, primiparous women had a higher likelihood of being provided epidural analgesia compared to multiparous women, and women with instrumental vaginal delivery had a higher likelihood compared to spontaneous delivery. This was expected, as primiparous women have a longer duration of delivery and more interventions by instrumental deliveries. In addition, instrumental vaginal delivery is more painful than spontaneous vaginal deliveries.Secondly, our results can be explained by premigration exposure to health system practices and norms in the home country. We found high provision of epidural analgesia among women born in Latin America/Caribbean region. In line with this, the epidural analgesia rate in Chile and in private health facilities in Brazil is higher than in Norway $[18,19]$. Conversely, in low- and middle-income countries, access to epidural analgesia is often suboptimal. In our study we found a low provision of epidural analgesia in women born in Sub-Saharan Africa. Outside tertiary facilities in these women's home countries management of labor pain often only involves non-pharmacological pain relief [20].

Thirdly, low uptake of pain relief in certain groups could be influenced by suboptimal communication, especially if language barriers were present. A study among 


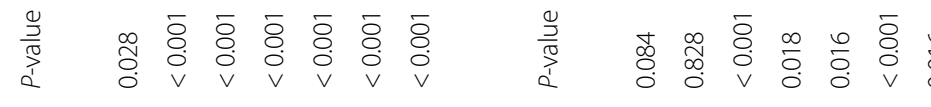

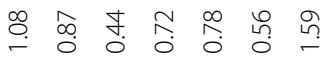

च̊

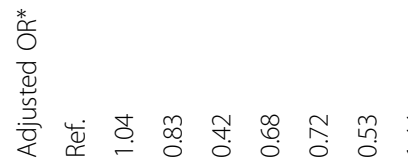

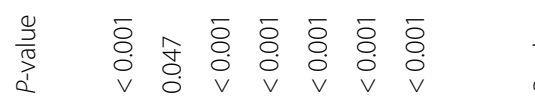

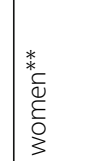

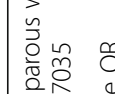

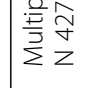

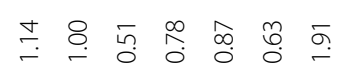

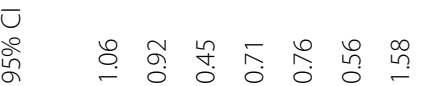

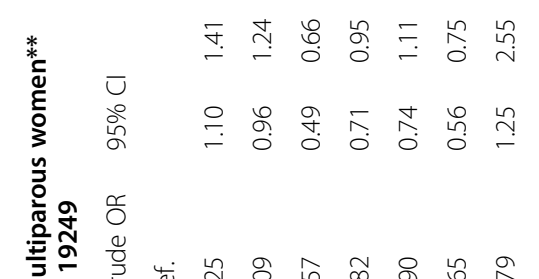

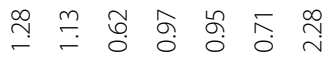

ত

范

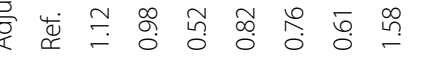

$\frac{\frac{9}{3}}{\frac{3}{2}}$

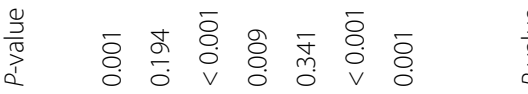

Ј

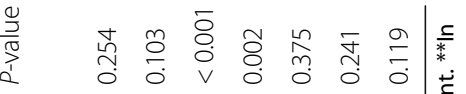

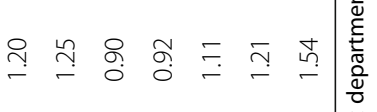

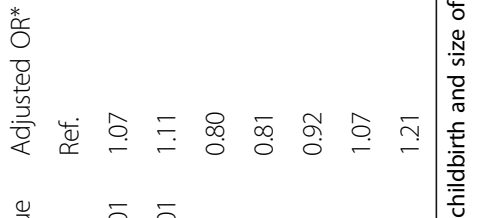

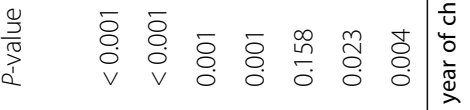

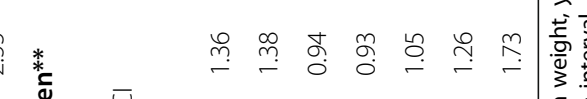

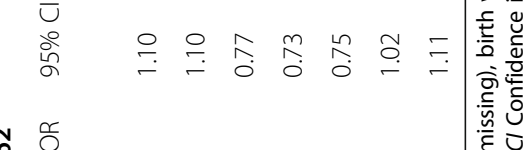

ํํำ

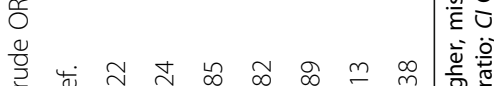

幽웅 \&

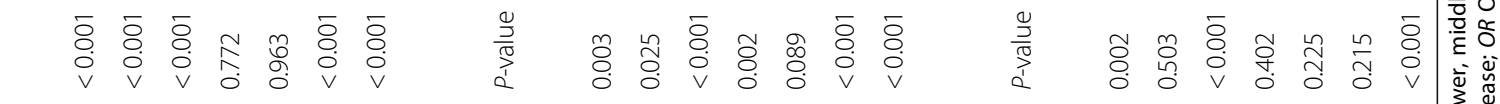

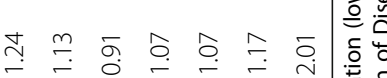

ঢ̄ं

㘶

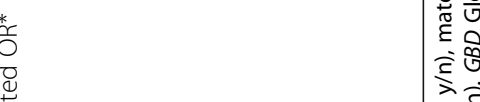

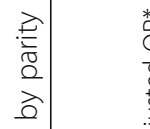

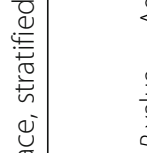

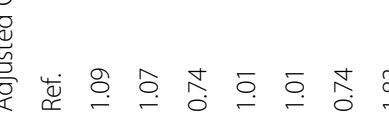

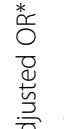

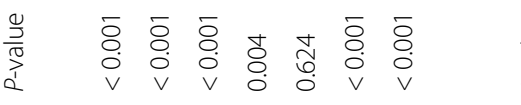

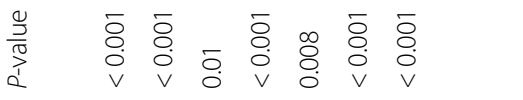

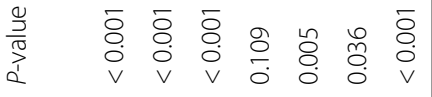

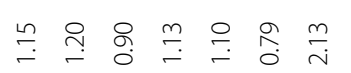

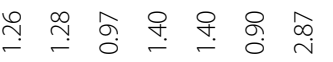

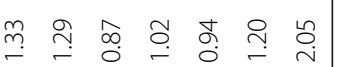

(2)

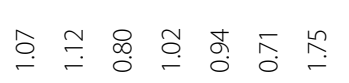

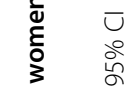

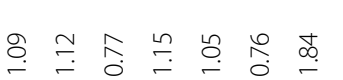

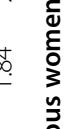

党

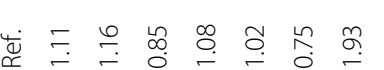

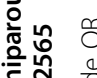

部

च

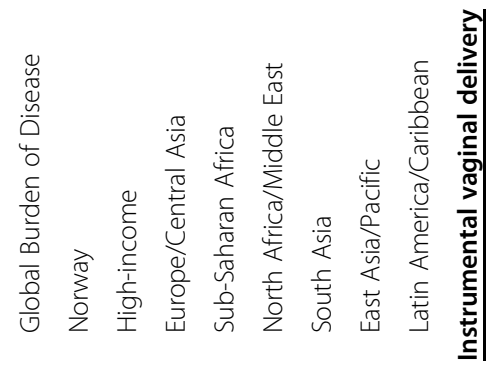

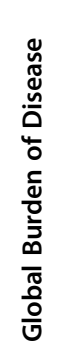

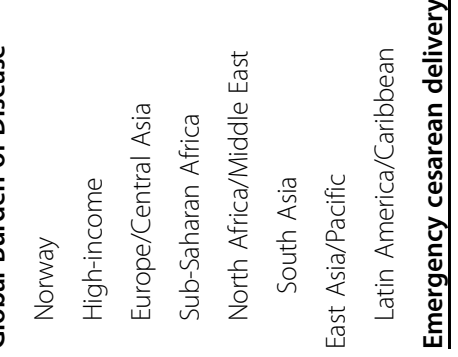

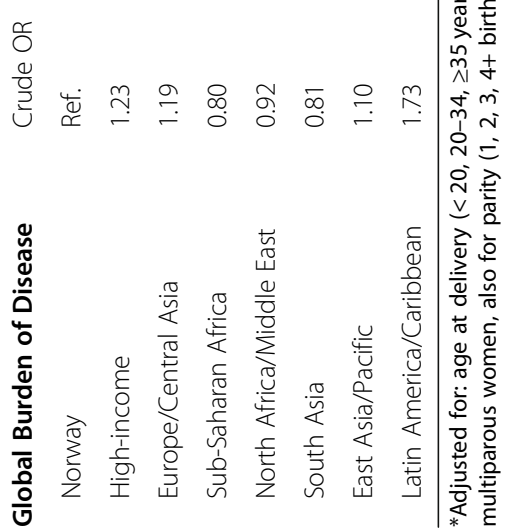



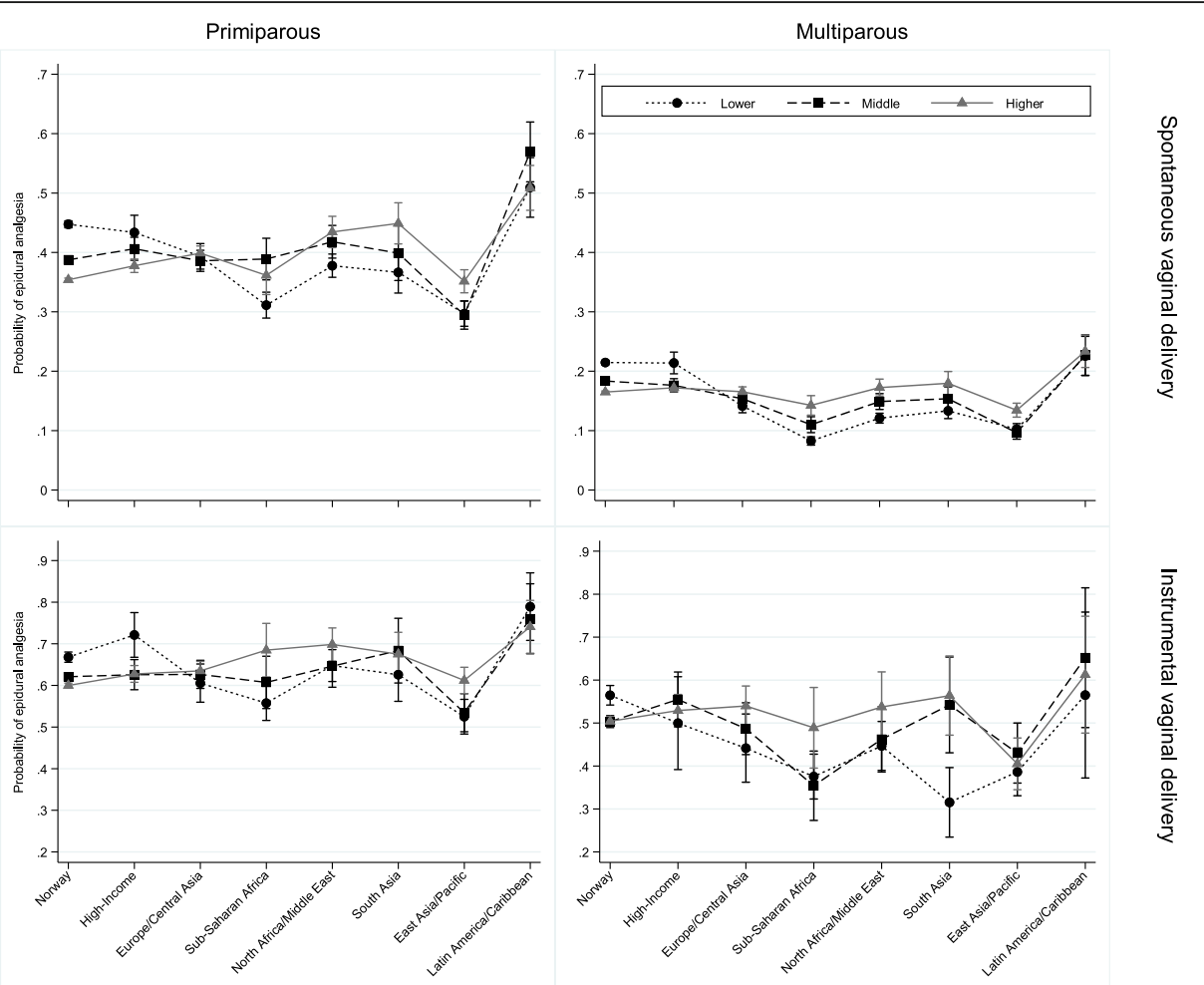

Fig. 1 Probability of epidural analgesia provision by maternal region of birth according to maternal education, stratified by mode of delivery (rows) and parity (columns). Maternal education; lower ( $\leq 10$ years), middle (11-13 years) and higher (> 13 years). Error bars: 95\% confidence intervals

Hispanic women in the US found lower provision of epidural analgesia among Hispanic women as compared to whites, and language barriers mediated that difference [21]. Both language barriers and misconceptions about possible pain relief may contribute to a communication barrier between the women and the health care providers [18]. Orejula et al. reported misconceptions about the safety of epidural analgesia in foreign-born women [18]. When language barriers are present, individual support by a laywoman (doula) matched by language and cultural preference of the woman giving birth, has been attempted with the aim of providing translation and advocacy to the woman [22]. A recent Cochrane review supports the use of doula as a resource to foreign-born delivering women in high-income countries [23].

Finally, health literacy and level of education could also have impacted our findings. Women in minority groups have previously reported poorer experience of maternity services [24]. We cannot exclude that lower provision of epidural may be determined by a paternalistic attitude among the healthcare staff towards women of lower socioeducational groups. Higher educated women born in Sub-Saharan Africa, North Africa/Middle East or South Asia were more likely to be provided epidural analgesia, compared to those with lower education from the same areas. Furthermore, we found increased provision of epidural analgesia with longer residence in Norway, implying a potential acculturation effect [25]. In our study, Pakistani born women constituted $72 \%$ of the South Asian group, which also was the group of women that had the longest residence time in Norway in our study. Longer residence time is associated with improved health literacy, including improved language proficiency, which could strengthen the participation in decision-making. Good language skills could modify a negative impact of ethnicity on the provision of analgesia during delivery [21]. At the same time, increasing familiarity with and knowledge of cultural-specific attitudes might improve the effort and communication skills among health staff themselves.

We also examined pain relief in emergency cesarean deliveries. In Norway, $21 \%$ of all cesarean deliveries are due to failure to progress [26], and in these women epidural analgesia is especially useful. However, as we lacked data on indication for emergency cesarean delivery, these results are difficult to interpret.

\section{Strengths and limitations}

Strengths of this study include the use of a large, nationwide birth cohort with minimal selection bias, including 
more than 175,000 births among immigrant women. The completeness of the MBRN is close to $100 \%$ and misclassifications are believed to be minimal $[14,15]$. The linkage with national statistics enabled us to include information on maternal education level and residence time in Norway, as proxy indicators of health literacy and acculturation, respectively. The use of the GBD framework to classify the immigrant population may also be evaluated as strength, as the framework combines geographical and economical aspects of the country of birth.

The study has some limitations. The categorization of different countries into GBD groups may cause a loss of detailed information from particular countries. In addition, we assume that women originating from a particular geographical region share common traits, disregarding the heterogeneity in sociocultural background, religion, attitudes and a selection to migration. We controlled for predefined potential confounders; however, we did not have information on language skills, interpreter use or health literacy. The relative risk of epidural analgesia was based on logistic regression analyses adjusted for potential confounders, assuming additive effects. When investigating the presence of effect modification, using a strict significance level, we found a clear and consistent interaction between the provision of epidural analgesia and education. However, due to the large sample size, we cannot exclude spurious interaction effects and results should be interpreted with caution. In addition, there has been an increased provision of epidural over the study time period. To take into any consideration time-dependent effects, we included year of birth in the regression analysis. Thus, we believe that any bias from time-dependent effects have been adjusted for in the final models.

Other types of pain relief (nitrous oxide, intravenous opiates etc) were outside the scope of this study, however we performed sub analyses for spinal analgesia and pudendal block (Supplementary Table 4 and 5).

Due to the heterogeneity of the immigrant populations, our results cannot necessarily be generalized to other settings. However, in countries with a similar immigration pattern and universal free maternity care, results may be similar. To determine why there are disparities in the provision of epidural analgesia, future studies exploring women's own perspectives are needed.

\section{Conclusion}

In this study, maternal birthplace was associated with the likelihood of being provided epidural analgesia. Further investigations, quantitative as well as qualitative, may help elucidate reasons for this diversity and provide knowledge about women's own needs and wishes.

\section{Supplementary information}

Supplementary information accompanies this paper at https://doi.org/10. 1186/s12884-020-03021-8.

\section{Additional file 1.}

Abbreviations

GBD: Global Burden of Disease; MBRN: Medical Birth Registry of Norway

\section{Acknowledgements}

Not applicable.

\section{Authors' contributions}

ÅHW planned the study in cooperation with the co-authors, analyzed and interpreted the data and wrote the manuscript. AFJ, ML, ACS, RSF, SV and IKS contributed substantially to the planning of the study, interpretation of the data and revision of the manuscript. RSF also contributed to analyzing and making the figure. All authors contributed to the submitted manuscript version. All authors have read and approved the manuscript.

\section{Funding}

This work was supported by a PhD salary from Oslo University Hospital, Norway.

\section{Availability of data and materials}

The anonymous datasets used and/or analyzed during the current study are available from the corresponding author on reasonable request.

Ethics approval and consent to participate

The study was approved by the Regional Committees for Medical and Health Research Ethics South East Norway in 2017 (reference 2016/417/REK) including waiver of participant's individual consent.

\section{Consent for publication}

Not Applicable.

\section{Competing interests}

The authors declare that they have no competing interests.

\section{Author details}

'Division of Obstetrics and Gynaecology, Oslo University Hospital, Sognsvannsveien 20, 0372 Oslo, Norway. ${ }^{2}$ Institute of Clinical Medicine, University of Oslo, Oslo, Norway. ${ }^{3}$ Institute of Health Sciences, Oslo Metropolitan University, Oslo, Norway. ${ }^{4}$ Institute of Health and Social Sciences, University of South-Eastern Norway, Campus Vestfold, Borre, Norway. ${ }^{5}$ Oslo Centre for Biostatistics and Epidemiology, Oslo University Hospital, Oslo, Norway. ${ }^{6}$ Norwegian National Advisory Unit on Women's Health, Oslo University Hospital, Oslo, Norway.

Received: 20 February 2020 Accepted: 19 May 2020

Published online: 26 May 2020

\section{References}

1. WHO recommendations. Intrapartum care for a positive childbirth experience. Geneva: World Health Organization Copyright (c) World Health Organization 2018; 2018.

2. Jones L, Othman M, Dowswell T, Alfirevic Z, Gates S, Newburn M, et al. Pain management for women in labour: an overview of systematic reviews. Cochrane Database Syst Rev. 2012;3:CD009234.

3. Karlsdottir SI, Sveinsdottir H, Kristjansdottir H, Aspelund T, Olafsdottir OA. Predictors of women's positive childbirth pain experience: findings from an Icelandic national study. Women Birth. 2018;31(3):e178-e84.

4. Swift EM, Tomasson G, Gottfresdottir H, Einarsdottir K, Zoega H. Obstetric interventions, trends, and drivers of change: a 20-year population-based study from Iceland. Birth. 2018;45(4):368-76.

5. Coylewright M, Montori $\mathrm{V}$, Ting HH. Patient-centered shared decisionmaking: a public imperative. Am J Med. 2012;125(6):545-7.

6. Charles C, Gafni A, Whelan T. Shared decision-making in the medical encounter: what does it mean? (or it takes at least two to tango). Soc Sci Med. 1997;44(5):681-92. 
7. Bae JM. Shared decision making: relevant concepts and facilitating strategies. Epidemiol Health. 2017;39:e2017048.

8. Vangen S, Stoltenberg C, Schei B. Ethnicity and use of obstetrical analgesia: do Pakistani women receive inadequate pain relief in labour? Ethnicity Health. 1996;1 (2):161-7.

9. OECD, Union E. Indicators of Immigrant Integration 20152015.

10. Bakken KS, Skjeldal OH, Stray-Pedersen B. Immigrants from conflict-zone countries: an observational comparison study of obstetric outcomes in a low-risk maternity ward in Norway. BMC Pregnancy Childbirth. 2015;15:163.

11. Sorbye IK, Stoltenberg C, Sundby J, Daltveit AK, Vangen S. Stillbirth and infant death among generations of Pakistani immigrant descent: a population-based study. Acta Obstet Gynecol Scand. 2014;93(2):168-74.

12. Sorbye IK, Daltveit AK, Sundby J, Vangen S. Preterm subtypes by immigrants' length of residence in Norway: a population-based study. BMC Pregnancy Childbirth. 2014;14:239.

13. Sorbye IK, Daltveit AK, Sundby J, Stoltenberg C, Vangen S. Caesarean section by immigrants' length of residence in Norway: a population-based study. Eur J Pub Health. 2015;25(1):78-84.

14. Lehmann S, Baghestan E, Bordahl P, Ebbing M, Irgens L, Rasmussen S, Validation of data in the medical birth registry of Norway on delivery after a previous cesarean section. Acta Obstet Gynecol Scand. 2017;96(7):892-7.

15. Baghestan E, Bordahl PE, Rasmussen SA, Sande AK, Lyslo I, Solvang I. A validation of the diagnosis of obstetric sphincter tears in two Norwegian databases, the medical birth registry and the patient administration system. Acta Obstet Gynecol Scand. 2007;86(2):205-9.

16. Global Burden of Disease Study 2015 (GBD 2015) Location Hierarchies Global Health Data Exchange [2018.09.19]. Available from: http://ghdx. healthdata.org/record/ihme-data/gbd-2015-location-hierarchies.

17. Klomp T, de Jonge A, Hutton EK, Lagro-Janssen AL. Dutch women in midwife-led care at the onset of labour: which pain relief do they prefer and what do they use? BMC Pregnancy Childbirth. 2013;13:230.

18. Orejuela FJ, Garcia T, Green C, Kilpatrick C, Guzman S, Blackwell S. Exploring factors influencing patient request for epidural analgesia on admission to labor and delivery in a predominantly Latino population. J Immigr Minor Health. 2012;14(2):287-91.

19. Ramires de Jesus G, Ramires de Jesus N, Peixoto-Filho FM, Lobato G. Caesarean rates in Brazil: what is involved? Obstet Anesthesia Digest. 2015; 1(5):606-9.

20. Akadri AA, Odelola Ol. Labour pain perception: experiences of Nigerian mothers. Pan Afr Med J. 2018;30:288.

21. Caballero JA, Butwick AJ, Carvalho B, Riley ET. Preferred spoken language mediates differences in neuraxial labor analgesia utilization among racial and ethnic groups. Int. 2014;23(2):161-7.

22. Mottl-Santiago J, Walker C, Ewan J, Vragovic O, Winder S, Stubblefield P. A hospital-based doula program and childbirth outcomes in an urban, multicultural setting. Matern Child Health J. 2008;12(3):372-7.

23. Bohren MA, Berger BO, Munthe-Kaas H, Tuncalp O. Perceptions and experiences of labour companionship: a qualitative evidence synthesis. Cochrane Database Syst Rev. 2019;3:CD012449.

24. Henderson J, Gao H, Redshaw M. Experiencing maternity care: the care received and perceptions of women from different ethnic groups. BMC Pregnancy Childbirth. 2013;13:196

25. Hawkins SS, Lamb K, Cole TJ, Law C. Millennium cohort study child health $\mathrm{G}$. influence of moving to the UK on maternal health behaviours: prospective cohort study. BMJ. 2008;1(7652):1052-5.

26. Kolas $T$, Hofoss $D$, Daltveit AK, Nilsen $S T$, Henriksen $T$, Hager $R$, et al. Indications for cesarean deliveries in Norway. Am J Obstet Gynecol. 2003; 188(4):864-70

\section{Publisher's Note}

Springer Nature remains neutral with regard to jurisdictional claims in published maps and institutional affiliations.

\section{Ready to submit your research? Choose BMC and benefit from:}

- fast, convenient online submission

- thorough peer review by experienced researchers in your field

- rapid publication on acceptance

- support for research data, including large and complex data types

- gold Open Access which fosters wider collaboration and increased citations

- maximum visibility for your research: over $100 \mathrm{M}$ website views per year

At BMC, research is always in progress.

Learn more biomedcentral.com/submissions 